\title{
Distribución de la pobreza energética en la ciudad de Madrid (España)
}

Fernando Martín-Consuegra. Universidad Politécnica de Madrid, Madrid, España. Agustín Hernández-Aja. Universidad Politécnica de Madrid, Madrid, España. Ignacio Oteiza. Consejo Superior de Investigaciones Científicas (CSIC), Madrid, España. Carmen Alonso. Consejo Superior de Investigaciones Científicas (csic), Madrid, España.

RESUMEN | Se desarrolla una metodología que permite la localización de áreas urbanas que se encuentran en situación de pobreza energética. Para ello es necesario analizar datos geolocalizados de la relación entre los ingresos medios por hogar y los consumos energéticos residenciales para cada área urbana. La metodología se aplica al caso de la ciudad de Madrid (España). En el proceso de estimación del coste de los servicios energéticos por hogar se desentrama el peso que juegan los distintos factores que influyen en él: la calidad de la construcción, las instalaciones térmicas más utilizadas, los tipos de suministro de energía empleados para satisfacer las necesidades del hogar y la estructura tarifaria del sector energético español. Se concluye que la interacción de estos factores provoca que los hogares incluidos en los tramos de renta mínima estén en riesgo de pobreza energética de manera independiente de la eficiencia energética de la vivienda que ocupan. Esto apunta a la necesidad de desarrollar políticas públicas específicas para combatir la pobreza energética de manera complementaria a los planes para la rehabilitación energética de viviendas.

PALABRAS CLAVE | indicadores ambientales, renovación urbana, vulnerabilidad.

ABSTRACT | In this article, we develop a methodology that allows the localization of urban areas that are suffering from fuel poverty. For this, we analyze average incomes per household and residential energy consumption for each urban area with Geographical Information Systems. This methodology is applied to a case study: The city of Madrid (Spain). By estimating the cost of energy services per household, the weight of the different factors influencing the energy services is uncovered: quality of construction, thermal facilities, energy sources supply and billing structure used by Spanish energy sector. We conclude that the interaction of these factors causes households-included in the minimum income brackets- to be at risk of energy poverty, despite of the energy efficiency of the unit. This means that specific public policies are needed to combat energy poverty, in order to complement energy refurbishment plans.

KEYWORDS | environmental indicators, urban renewal, vulnerability.

Recibido el 11 de octubre de 2017, aprobado el 5 de julio de 2018.

E-mails: M. Martín-Consuegra, martin-consuegra@ietcc.csic.es | A. Hernández-Aja, agustin.hernandez@upm.es | I. Oteiza, ioteiza@ietcc. csic.es | C. Alonso, c.alonso@ietcc.csic.es 


\section{Introducción}

La pobreza energética se produce por la incapacidad de mantener un hogar en condiciones de confort adecuadas para los estándares actuales (Comité Económico y Social Europeo [CESE], 2011). Ella resulta principalmente de la combinación de tres factores: bajo nivel de renta, calidad de la edificación insuficiente y elevados precios de la energía (Thomson \& Snell, 2013). Se trata de un problema social, medioambiental y de salud pública que parece destinado a agravarse en Europa en los próximos tiempos (European Commission \& Directorate-General for Energy, 2010).

El sur de Europa padece los niveles más altos de pobreza energética del continente y las condiciones más precarias de vivienda (Healy, 2003). El exceso de mortalidad en ciertas estaciones del año es algo que se debe tener muy en cuenta, pues si analizamos la pobreza energética como parte de su causa, se podría reducir con solo adecuar las viviendas a unas mejores condiciones (wwF, Velázquez, Verdaguer, Sanz \& Gómez, 2017). Estudios recientes señalan también el sur de Europa como la zona donde se concentran los países en los que se han reportado mayores niveles de instalaciones de calefacción inadecuadas en los hogares y falta de aislamiento térmico en la edificación, con especial incidencia en Portugal, Malta y Espańa (Lelkes \& Zólyomi, 2010, 2015).

En el caso de España, la calidad térmica de la envolvente de la mayor parte del parque residencial edificado se ha demostrado insuficiente (Oteiza, Alonso, MartínConsuegra, González-Moya \& Monjo, 2015). Este parque requiere de una rehabilitación integral si se pretende alcanzar los objetivos de las directivas europeas en materia de eficiencia energética y cambio climático (Parlamento Europeo \& Consejo de la Unión Europea, 2012). En 2014, el 10\% de las familias no podía mantener su casa en condiciones adecuadas de temperatura durante el invierno (Tirado Herrero et al., 2016), de acuerdo con el indicador LiHc (Low Income-High Cost) (Hills, 2012). Las consecuencias son una pérdida de calidad de vida, falta de confort y problemas de salud asociados mayoritariamente a personas débiles a las cuales, en el peor de los casos, puede producirles la muerte (Liddell \& Morris, 2010).

El presente estudio se apoya en un trabajo previo en el cual se realizó la caracterización de la calidad térmica de la edificación residencial a partir de la estimación de la demanda energética necesaria para lograr el confort en invierno para todas las viviendas principales de la ciudad de Madrid (Martín-Consuegra, Hernández Aja, Oteiza \& Alonso, 2016). Este estudio, en sintonía con otros estudios sobre la eficiencia energética de la edificación española (Cuchí, Sweatman \& Rehabilitation Working Group, 2012; Dalle et al., 2010; Oteiza, Alonso, Martín-Consuegra \& Buldón, 2016), concluye que las elevadas necesidades de energía de estas viviendas podrían verse muy reducidas mediante su rehabilitación energética. Las secciones censales se clasifican conforme a su demanda de calefacción (figura 1), con una demanda de energía para calefacción muy elevada de $123.65 \mathrm{kWh} / \mathrm{m}^{2}$, lo que se correspondería con una etiqueta de clasificación energética $\mathrm{F}$. 


\section{Objeto del estudio}

El objetivo general es la estimación de un indicador de pobreza energética desarrollado a partir del cálculo de la demanda energética para calefacción de la edificación residencial y del nivel de renta medio de los hogares para el municipio de Madrid. El ámbito de estudio está constituido por las áreas urbanas. Los datos se utilizan a escala inframunicipal, con la máxima desagregación posible: la sección censal. Las secciones son un referente geográfico de carácter estadístico y constituyen un instrumento eficaz tanto en trabajos censales o padronales, como para investigaciones por muestreo y en el Censo Electoral. Se desarrolla el indicador de pobreza energética con el objeto de incluirlo en el conjunto de indicadores sintéticos a partir de los que es posible delimitar ámbitos de vulnerabilidad integral, llamados barrios vulnerables (Temes, 2014).

El enfoque de este trabajo se dirige principalmente al consumo de energía en calefacción, que supone el 55\% del consumo energético de los hogares de la zona continental mediterránea (Instituto para la Diversificación y Ahorro de Energía [IDAE], 2011b), en la que se encuentra la ciudad de Madrid. Se aborda también, de forma simplificada, el gasto correspondiente a otros consumos energéticos domésticos.

\section{Metodología}

En este trabajo se entiende la pobreza energética como la confluencia de factores que llevan a un consumo de energía que se considera excesivo en relación con la renta disponible en los hogares. Se localizan las secciones censales en las que el gasto en facturas energéticas de las viviendas principales supone más del 10\% de los ingresos anuales del hogar, de acuerdo con la primera definición de pobreza energética.

Para la estimación del riesgo de pobreza energética se ha utilizado el modelo de ingresos y gastos inspirado en el trabajo de Sánchez-Guevara Sánchez (2015), a partir del análisis de datos estadísticos y las características térmicas de la edificación. Para el estudio a la escala de barrio se comparan los datos de ingresos medios por hogar de cada sección censal (nivel de renta media), con el promedio del gasto equivalente en facturas energéticas en los hogares que contiene, estableciendo el umbral de la pobreza energética en el $10 \%$ de la renta.

\section{Demanda energética para calefacción}

La demanda representa las necesidades de energía de un recinto para conseguir las condiciones de confort que aparecen descritas y reglamentadas en la normativa vigente, en el caso de España a través del Código Técnico de la Edificación (CTEDB-HE, 2013). En el presente estudio, la calidad térmica de la edificación se define a partir de los valores para la demanda energética de calefacción que le corresponde a la envolvente térmica de cada periodo de construcción (Aksoezen, Hassler \& Kohler, 2015; Gómez Muñoz, 2014). Se clasifica cada sección censal conforme a una etiqueta energética, tomando para ello los valores límite de cada clase que se 
detallan en el procedimiento de calificación energética de los edificios existentes en España (IDAe, 2011a).

FIGURA I | Demanda de calefacción por sección censal en Madrid

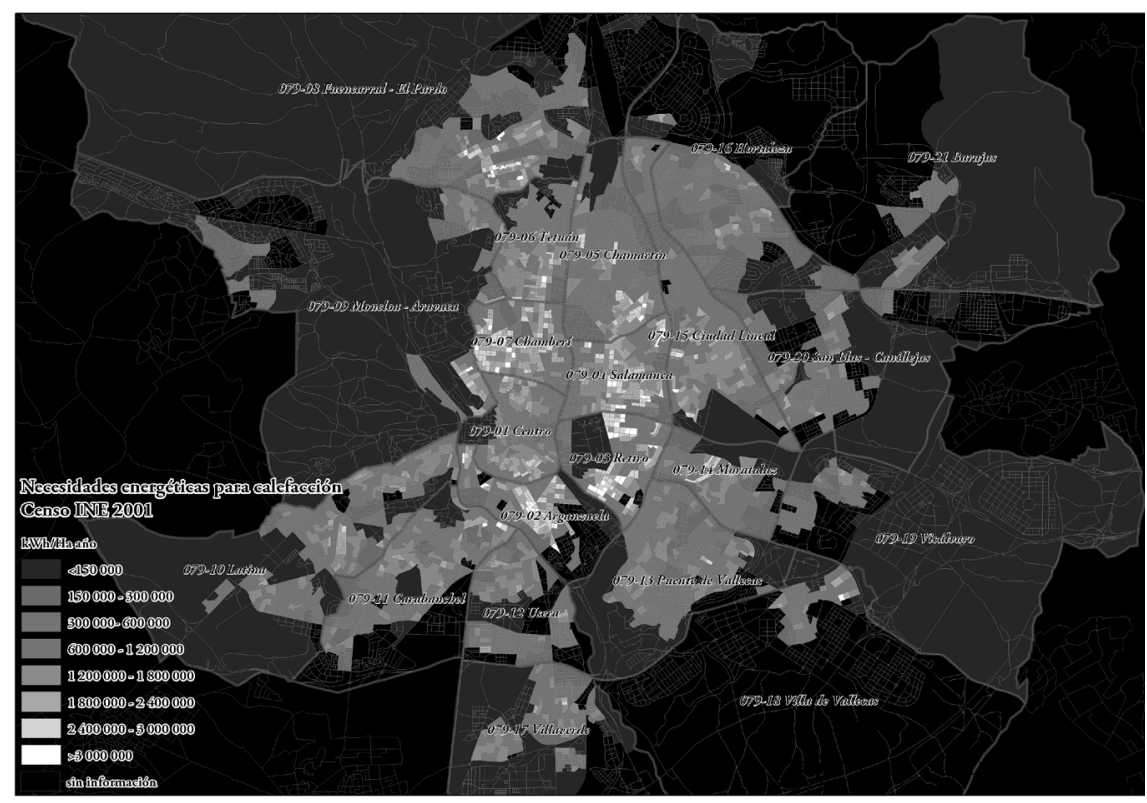

FUENTE MARTÍN-CONSUEGRA, HERNÁNDEZ AJA, OTEIZA Y ALONSO (20I6), P. I.4I7

En la ciudad de Madrid, las viviendas se organizan principalmente de forma colectiva en edificios multifamiliares. De acuerdo con el proyecto Sech-Spahousec (IDAE, 2011b), en las viviendas en bloque de la zona centro de España la calefacción es el consumo energético mayoritario, con el 55\% del consumo total por vivienda.

A partir de la demanda media de calefacción de cada clase, ha sido posible caracterizar las necesidades energéticas de calefacción de forma simplificada para todas las viviendas de una sección censal (figura 1). Con este indicador de demanda se asume que, durante el invierno, los hogares están a una temperatura adecuada y, por tanto, se evalúa la situación a partir de la suposición de que todos los habitantes tienen derecho a condiciones de confort mínimas.

\section{Consumo energético en calefacción estimado para la vivienda media en Madrid}

El consumo de calefacción se estima a partir de la demanda y el tipo de sistema utilizado. Para los casos en los que la fuente de energía es el gas natural, se ha considerado un rendimiento del $75 \%$ correspondiente al sistema más utilizado en la zona mediterránea continental de Espańa: la caldera individual (IDAE, 2011b). En el caso de que el sistema empleado para calefacción sea la electricidad por efecto Joule, se supone un rendimiento del $100 \%$ conforme al procedimiento de certificación energética de edificios existentes vigente (Efinovatic \& Centro Nacional de 
Energías Renovables [CENER], 2016) y, por tanto, en ese caso el consumo se iguala a la demanda. Para hacer una estimación de una vivienda habitual se ha utilizado el valor medio de superficie de vivienda en Madrid, de 80,76 $\mathrm{m}^{2}$ (Instituto Nacional de Estadística [INE], 2011). Con ello se obtiene que la demanda energética de calefacción de la vivienda media de Madrid es de $10.014 \mathrm{kWh}$ anuales $\left(124 \mathrm{kWh} / \mathrm{m}^{2}\right)$. Para cubrir esta demanda es necesario un consumo anual de $10.014 \mathrm{kWh}$ en los casos en que la fuente de energía es la electricidad, y de $13.252 \mathrm{kWh}$ en calefacciones de gas natural.

Siguiendo estos mismos pasos, es posible hallar una estimación aproximada de los consumos energéticos de calefacción que tendrían las diferentes calidades energéticas conforme a las distintas clases de demanda (para la vivienda de tamaño medio), siendo la clase a la más eficiente y la G la que más energía demanda. Se calcula el consumo energético (kWh) necesario para satisfacer la demanda energética para cada clase energética de la escala establecida en el procedimiento de certificación. En el caso de la clase energética G, no es posible utilizar un valor medio; en consecuencia, se ha utilizado el valor límite para caracterizar las viviendas que se encuentran en esa circunstancia, que son las de peor calidad térmica de la ciudad.

Coste de la energía para satisfacer la demanda de calefacción de las viviendas en Madrid

Para realizar una estimación del monto de facturación del consumo energético correspondiente al servicio de calefacción, es necesario tener en cuenta el gasto a partir del tipo de combustible empleado (figura $2 \mathrm{a}$ y $2 \mathrm{~b}$ ). De acuerdo con el Censo 2001 (INe, 2001), se comprueba (tabla 1) que casi el 60\% de los hogares de la ciudad de Madrid empleaba ya el gas natural, que resulta el combustible más económico en el momento del estudio. Un $20 \%$ de hogares emplea el suministro eléctrico para la calefacción y las fuentes de energía restantes (petróleo, madera, carbón) se consideran anecdóticas para una estimación gruesa.

TABLA I | Combustible usado para calefacción por número y porcentaje de hogares

\begin{tabular}{|c|r|r|r|r|r|r|c|}
\hline \multicolumn{1}{|c|}{ GAS } & $\begin{array}{c}\text { ELECTRI- } \\
\text { CIDAD }\end{array}$ & PETRÓLEO & MADERA & CARBÓN & OTROS & $\begin{array}{c}\text { NO } \\
\text { APLICABLE }\end{array}$ & TOTAL \\
\hline 640.321 & 220.904 & 144.224 & 3.808 & 41.837 & 4.368 & 24.844 & 1.080 .306 \\
\hline $59,27 \%$ & $20,45 \%$ & $13,35 \%$ & $0,35 \%$ & $3,87 \%$ & $0,40 \%$ & $2,30 \%$ & $100,00 \%$ \\
\hline
\end{tabular}

FUENTE INE, 200I 
FIGURA 2A | Fuentes de energía en los hogares del municipio de Madrid. Implantación de calefacción de gas

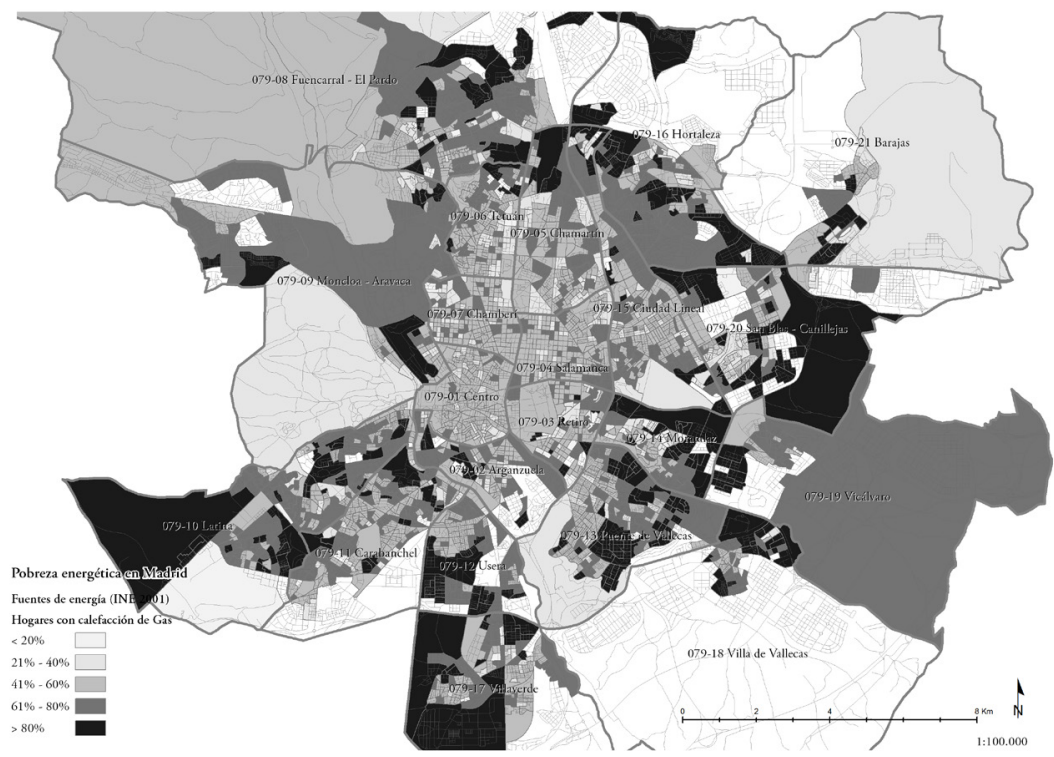

FUENTE ELABORACIÓN PROPIA A PARTIR DE DATOS INE, 200 I

FIGURA 2B $\mid$ Fuentes de energía en los hogares del municipio de Madrid. Implantación de calefacción eléctrica

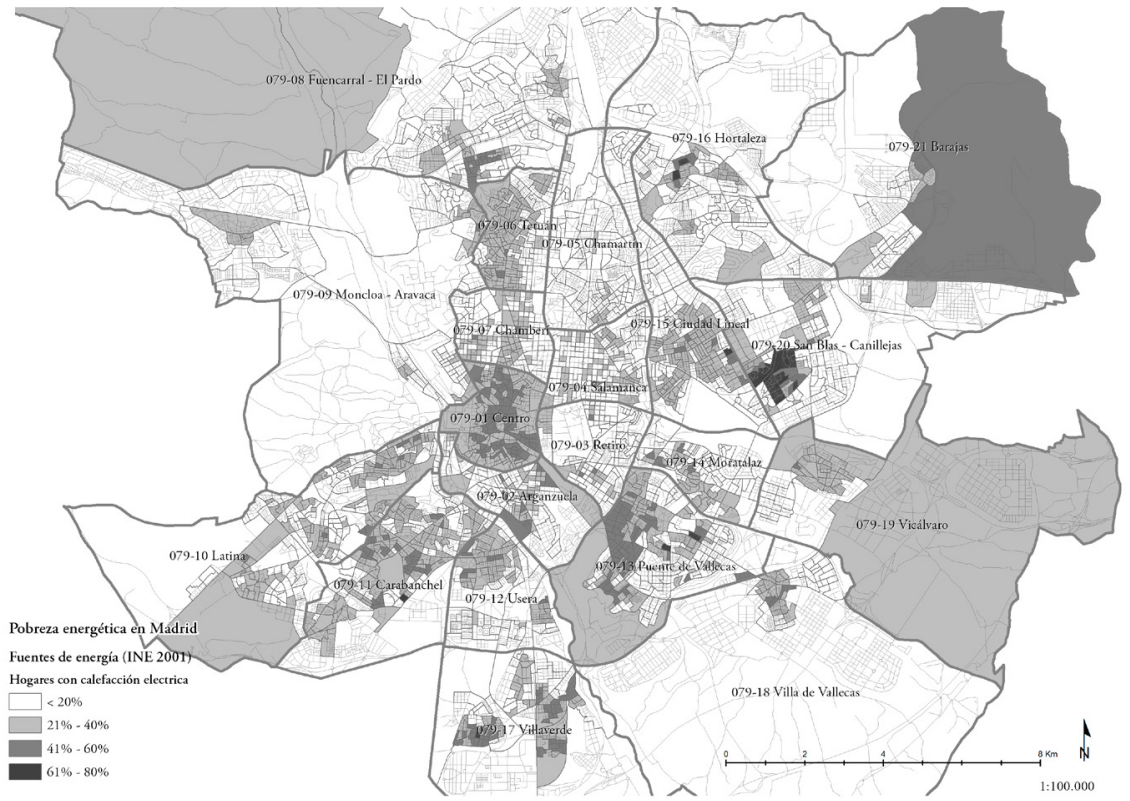

FUENTE ELABORACIÓN PROPIA A PARTIR DE DATOS INE, 200 I 
Para la estimación del coste que suponen los consumos en los hogares con calefacción de gas se ha utilizado el método de cálculo del coste descrito en Alonso, Martín-Consuegra y Lucas (2013). Dicho método se basa en la herramienta web Comparador de Ofertas de Energía: Gas y Electricidad, habilitada por la Comisión Nacional de los Mercados y la Competencia de España, que permite consultar las ofertas de gas natural disponibles para consumidores del grupo 3 (hasta 4 bares de presión) y ofertas de electricidad para consumidores en baja tensión (Comisión Nacional de los Mercados y la Competencia [CNM], 2017). Es importante señalar que el mercado español ha sido recientemente liberalizado y el precio de la energía varía en función de la empresa suministradora (García, 2006). En el caso de la electricidad se ha supuesto una potencia contratada de 3,30 kW, tarifa eléctrica normal sin discriminación horaria y sin servicios adicionales. Se comprueba que el precio de la electricidad varía mucho según la compañía que oferte el servicio (de 1.620,13€ a 2.015,78 € en el momento de la consulta: julio de 2017). A modo de ejemplo, se ha tomado el precio de una suministradora muy establecida en el mercado español.

TABLA 2 | Descomposición de la factura energética anual necesaria para el acondicionamiento térmico en invierno de una vivienda media en Madrid por tipo de suministro

\begin{tabular}{|l|c|c|c|c|c|c|c|}
\hline $\begin{array}{c}\text { TIPO DE } \\
\text { ENERGÍA }\end{array}$ & $\begin{array}{c}\text { CONSUMO } \\
(\mathbf{K W})\end{array}$ & $\begin{array}{c}\text { CONSUMO } \\
(\boldsymbol{\epsilon})\end{array}$ & $\begin{array}{c}\text { TÉRMINO } \\
\text { FIJO O DE } \\
\text { POTENCIA }(\boldsymbol{\epsilon})\end{array}$ & $\begin{array}{c}\text { IMPUESTO } \\
\text { HCLEC } \\
(\boldsymbol{(})\end{array}$ & $\begin{array}{c}\text { SUBTOTAL } \\
(\boldsymbol{\epsilon})\end{array}$ & $\begin{array}{c}\text { IVA 2 I \% } \\
(\boldsymbol{\epsilon})\end{array}$ & $\begin{array}{c}\text { TOTAL } \\
(\boldsymbol{\epsilon} / \text { AÑo) }\end{array}$ \\
\hline Gas & 13.352 & 597,21 & 101,40 & 31,24 & 729,86 & 31,24 & 883,13 \\
\hline Electricidad & 10.014 & $1.155,74$ & 135,82 & 66,03 & $1.357,59$ & 285,09 & $1.642,68$ \\
\hline
\end{tabular}

FUENTE ELABORACIÓN PROPIA, 2017

Se comprueba que el coste para mantener la vivienda en condiciones de confort empleando como combustible el gas natural sería de 883,13 €, es decir, aproximadamente 73,60 € mensuales si se reparte el gasto a lo largo de todo el año. En el caso de la electricidad el gasto es sensiblemente mayor, de al menos 1.642,68 €/año o $136,89 €$ mensuales (tabla 2).

Para asociar los consumos medios de cada sección censal y visualizar los datos mediante un Sistema de Información Geográfica (GIS), se calculan los costes de calefacción de cada sección censal conforme a las diferentes clases de la escala de calificación energética (tabla 3 a y 3 b). Los cálculos están realizados en todo momento para una vivienda de tamaño medio en Madrid (superficie de 80,76 $\mathrm{m}^{2}$ ). En ambos casos, las estimaciones indican que la estructura tarifaria española resulta en una repercusión del coste por unidad de energía suministrada más elevada cuanto más bajo es el consumo energético. Los hogares cuya vivienda está clasificada como clase A deben abonar un precio unitario por $\mathrm{kWh}$ que duplica aproximadamente el de la vivienda de clase G. 
TABLA 3A | Descomposición de la factura para calefacción por clases para la zona climática D (Madrid). Calefacción de gas

\begin{tabular}{|c|c|c|c|c|c|c|c|c|}
\hline CLASE & $\begin{array}{c}\text { CONSUMO } \\
(\mathrm{KWH})\end{array}$ & $\begin{array}{c}\text { CONSUMO } \\
(€)\end{array}$ & $\begin{array}{c}\text { TÉRMINO } \\
\text { FIJO (€) }\end{array}$ & $\begin{array}{c}\text { IMPUESTO } \\
\text { HC }(€)\end{array}$ & $\begin{array}{c}\text { SUBTOTAL } \\
(€)\end{array}$ & $\begin{array}{c}\text { IVA } 2 \text { I \% } \\
(€)\end{array}$ & $\begin{array}{c}\text { TOTAL } \\
(€ / \text { AÑo) }\end{array}$ & $\begin{array}{c}\text { COSTE } \\
\text { KWH } \\
(€ / \text { KWH })\end{array}$ \\
\hline A & 1.012 & 52,22 & 51,72 & 2,37 & 106,31 & 22,33 & 128,63 & 0,1271 \\
\hline B & 1.683 & 86,85 & 51,72 & 3,94 & 142,50 & 29,93 & 172,43 & 0,1025 \\
\hline C & 3.311 & 170,86 & 51,72 & 7,75 & 230,32 & 48,37 & 278,69 & 0,0842 \\
\hline $\mathrm{D}$ & 5.705 & 255,17 & 101,40 & 13,35 & 369,92 & 77,68 & 447,61 & 0,0785 \\
\hline $\mathrm{E}$ & 10.096 & 451,58 & 101,40 & 23,62 & 576,60 & 121,09 & 697,69 & 0,0691 \\
\hline F & 13.644 & 610,27 & 101,40 & 31,93 & 743,60 & 156,16 & 899,76 & 0,0659 \\
\hline G & 14.237 & 636,80 & 101,40 & 33,31 & 771,51 & 162,02 & 933,53 & 0,0656 \\
\hline
\end{tabular}

FUENTE ELABORACIÓN PROPIA, 2017

TABLA 3B | Descomposición de la factura para calefacción por clases para la zona climática D (Madrid). Calefacción eléctrica

\begin{tabular}{|c|c|c|c|c|c|c|c|c|}
\hline CLASE & $\begin{array}{c}\text { CONSUMO } \\
(\text { KWH })\end{array}$ & $\begin{array}{c}\text { CONSUMo } \\
(€)\end{array}$ & \begin{tabular}{|c|} 
TÉRMINO DE \\
POTENCIA \\
$(€)$
\end{tabular} & $\begin{array}{l}\text { IMPUESTO } \\
\text { ELEC }(€)\end{array}$ & $\begin{array}{c}\text { SUBTOTAL } \\
(€)\end{array}$ & $\begin{array}{c}\text { IVA } 2 \text { I \% } \\
(€)\end{array}$ & $\begin{array}{c}\text { TOTAL } \\
\text { (€/AÑo) }\end{array}$ & $\begin{array}{c}\text { COSTE } \\
\text { KWH }(€ / \\
\text { KWH }) \\
\end{array}$ \\
\hline A & 759 & 87,60 & 135,82 & 11,42 & 234,84 & 49,32 & 284,15 & 0,3744 \\
\hline B & 1.262 & 145,65 & 135,82 & 14,39 & 295,86 & 62,12 & 357,99 & 0,2837 \\
\hline $\mathrm{C}$ & 2.483 & 286,57 & 135,82 & 21,60 & 442,98 & 93,24 & 537,22 & 0,2164 \\
\hline $\mathrm{D}$ & 4.279 & 493,85 & 135,82 & 32,19 & 661,86 & 138,99 & 800,85 & 0,1872 \\
\hline E & 7.572 & 873,90 & 135,82 & 51,62 & $1.061,34$ & 222,88 & $1.284,22$ & 0,1696 \\
\hline $\mathrm{F}$ & 10.233 & $1.181,01$ & 135,82 & 67,33 & $1.384,15$ & 290,67 & $1.674,82$ & 0,1637 \\
\hline G & 10.678 & $1.232,37$ & 135,82 & 69,95 & $1.438,14$ & 302,01 & $1.740,15$ & 0,1630 \\
\hline
\end{tabular}

FUENTE ELABORACIÓN PROPIA, 2017

En la zona climática continental de España, en la que se encuentra Madrid, los sistemas de calefacción centralizada en edificios multifamiliares están presentes en el 18\% de los hogares (IDAE, 2011b). Dichos sistemas presentan mejores rendimientos que los de la caldera individual, lo que reduciría los costes por consumo energético. Las instalaciones de calefacción por distrito presentan balances todavía más efectivos en la gestión de los recursos energéticos, reduciéndose aún más estos costes (Song, Li \& Wallin, 2017). La implantación de estos últimos en la ciudad de Madrid se reduce a unos pocos casos anecdóticos, siendo conocidas las redes de la Ciudad Universitaria de Madrid, la Colonia de la Esperanza, el Barrio Altamira y la Meseta de Orcasitas. En el presente estudio, para simplificar la metodología, las instalaciones colectivas no han sido tenidas en cuenta de forma diferenciada, utilizándose el mismo rendimiento para todas las instalaciones de gas. Esta simplificación no impide conseguir el objetivo de desvelar los casos más críticos. 


\section{Estimación del consumo total}

La metodología para la estimación de la pobreza energética empleada hace referencia al porcentaje de ingresos del hogar que representa el gasto total en facturas energéticas, y no solo el gasto en calefacción. Para comparar los valores de renta con la factura, es necesario estimar cuál sería su coste total añadiendo el resto de servicios. Además del $55 \%$ de la energía utilizado en la calefacción para la zona centro, el resto de consumos se refiere a electrodomésticos (17,6\%), Agua Caliente Sanitaria (ACs) $(17,4 \%)$, cocina $(6,5 \%)$, iluminación $(2,6 \%)$ y aire acondicionado $(0,7 \%)$.

Para el cálculo del consumo energético total, se añade al coste del suministro de energía para calefacción al del resto de los servicios, conforme a las estimaciones del IDAE $(4403,7 \mathrm{kWh})$. Para estos usos se ha considerado, de forma simplificada para todos los casos, que se utiliza la energía eléctrica. Estos valores se suman al consumo de energía para calefacción. Se entiende que en el caso de las viviendas con calefacción eléctrica no procede computar por segunda vez la cantidad correspondiente al término de potencia y, por lo tanto, se ha sumado únicamente en la estimación de la factura correspondiente a las viviendas que se calientan por gas. El gasto correspondiente a otros usos sería de 819,20 €/año para la vivienda con calefacción de gas natural y de $646,43 €$ ańo para la que tiene calefacción eléctrica.

Sumando los gastos de calefacción (tabla 2) a los del resto de usos, se obtiene una aproximación a los consumos energéticos totales anuales para una vivienda media de Madrid (clase de calefacción F y superficie de $80,76 \mathrm{~m}^{2}$ ). Estos gastos serían de $1.702,33 €$ en viviendas con calefacción de gas y de 2.289,11 $€$ en viviendas con calefacción eléctrica. De la misma forma, resulta posible calcular los costes totales, tanto energético como económico, para la vivienda media de cada clase de demanda de calefacción (tabla 4). Se obtiene a partir de la adición del coste correspondiente a "resto de usos", al coste de suministro energético para calefacción calculado anteriormente.

TABLA 4 Consumo total anual por vivienda por clase de demanda de calefacción según fuente de energía (1) gas y (2) eléctrica, para la zona climática D2 (Madrid)

\begin{tabular}{|c|c|c|c|c|c|c|c|c|c|c|c|c|}
\hline \multicolumn{2}{|c|}{$\begin{array}{c}\text { CALEFACCIÓN } \\
\text { DE GAS }\end{array}$} & \multicolumn{2}{|c|}{ OTROS USOS } & \multicolumn{2}{|c|}{ TOTAL } & \multirow{2}{*}{$\begin{array}{c}\text { CLASE } \\
\text { CAL }\end{array}$} & \multicolumn{2}{|c|}{$\begin{array}{l}\text { CALEFACCIÓN } \\
\text { ELÉCTRICA }\end{array}$} & \multicolumn{2}{|c|}{ OTROS USOS } & \multicolumn{2}{|c|}{ TOTAL } \\
\hline KWH & $€$ & KWH & $€$ & KWH & $€$ & & KWH & $€$ & KWH & $€$ & KWH & $€$ \\
\hline 1.012 & 129 & 4.404 & 819 & 5.416 & 948 & A & 759 & 284 & 4.404 & 646 & 5.163 & 931 \\
\hline 1.683 & 172 & 4.404 & 819 & 6.087 & 992 & B & 1.262 & 358 & 4.404 & 646 & 5.666 & 1.004 \\
\hline 3.311 & 279 & 4.404 & 819 & 7.715 & 1.098 & $\mathrm{C}$ & 2.483 & 537 & 4.404 & 646 & 6.887 & 1.184 \\
\hline 5.705 & 448 & 4.404 & 819 & 10.109 & 1.267 & $\mathrm{D}$ & 4.279 & 801 & 4.404 & 646 & 8.683 & 1.447 \\
\hline 10.096 & 698 & 4.404 & 819 & 14.500 & 1.517 & $\mathrm{E}$ & 7.572 & 1.284 & 4.404 & 646 & 11.976 & 1.931 \\
\hline 13.644 & 900 & 4.404 & 819 & 18.048 & 1.719 & $\mathrm{~F}$ & 10.233 & 1.675 & 4.404 & 646 & 14.637 & 2.321 \\
\hline 14.237 & 934 & 4.404 & 819 & 18.641 & 1.753 & G & 10.678 & 1.740 & 4.404 & 646 & 15.082 & 2.387 \\
\hline
\end{tabular}




\section{FIGURA 3 | Coste anual del consumo energético residencial medio por sección} censal

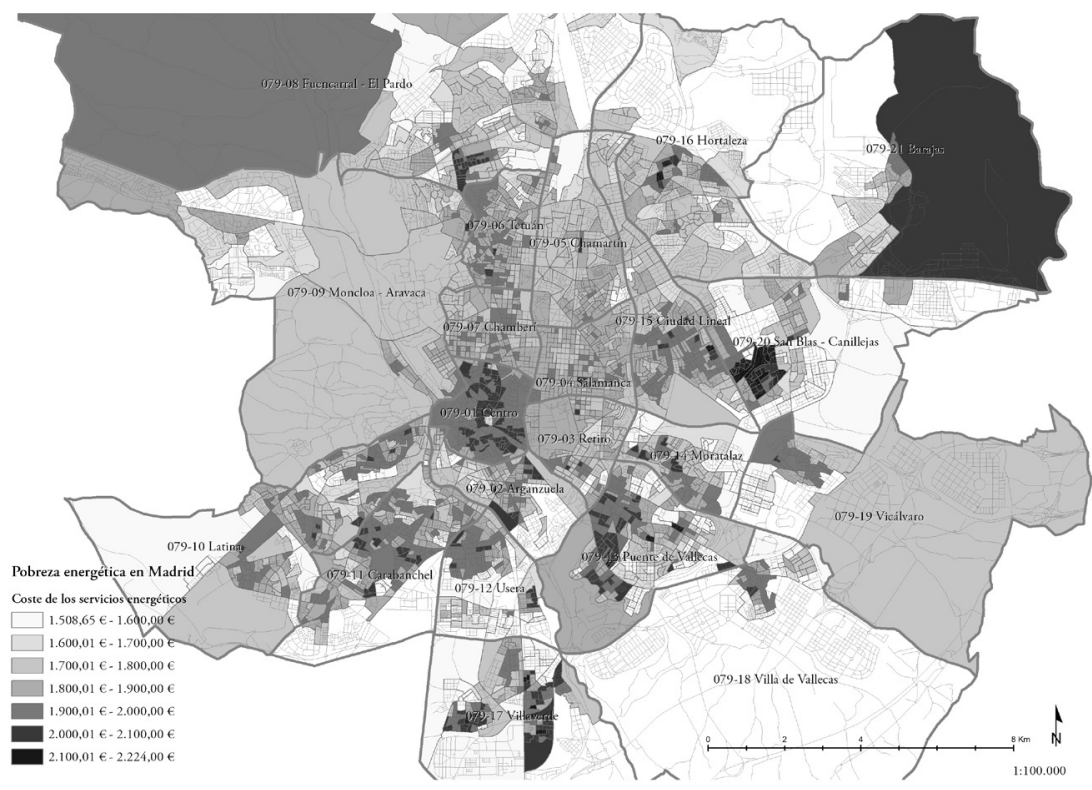

FUENTE ELABORACIÓN PROPIA, 2017

Para la estimación del coste del consumo energético total para todas las viviendas de cada sección censal (figura 3), se ha tenido en cuenta la proporción de viviendas con calefacción eléctrica y gas natural. Las restantes fuentes de energía se han asimilado, de forma simplificada, a la del gas natural.

\section{Nivel de renta}

La información utilizada acerca de la renta neta media anual de los hogares por sección censal ha sido facilitada por el Ayuntamiento (Ayuntamiento de Madrid, 2017). Esta información es fruto de la colaboración del Instituto Nacional de Estadística (INE) con la Agencia Española de Administración Tributaria (AEAT) para la utilización de datos tributarios con fines estadísticos. El estudio tiene en cuenta dos componentes: la renta neta media anual (AEAT) y el número de hogares a partir de una explotación del Padrón Continuo (INE). La recopilación de datos se ha realizado para el proyecto Urban Audit (European Environment Agency, 2017) siguiendo la metodología descrita en el manual de Estadística Urbana de Eurostat (European Union, 2017) y se distribuyen en diez tramos de renta (figura 4). En el tramo más bajo se han incluido estimaciones para los valores de renta correspondientes al Salario Mínimo Interprofesional (sMI) descrito por el Ministerio de Empleo y Seguridad Social (EmpleoGob, 2015) y a la Renta Mínima de Inserción (RMI) definida para la Comunidad Autónoma de Madrid (CAM, 2017). 
FIGURA 4 Distribución de la renta por tramos en Madrid

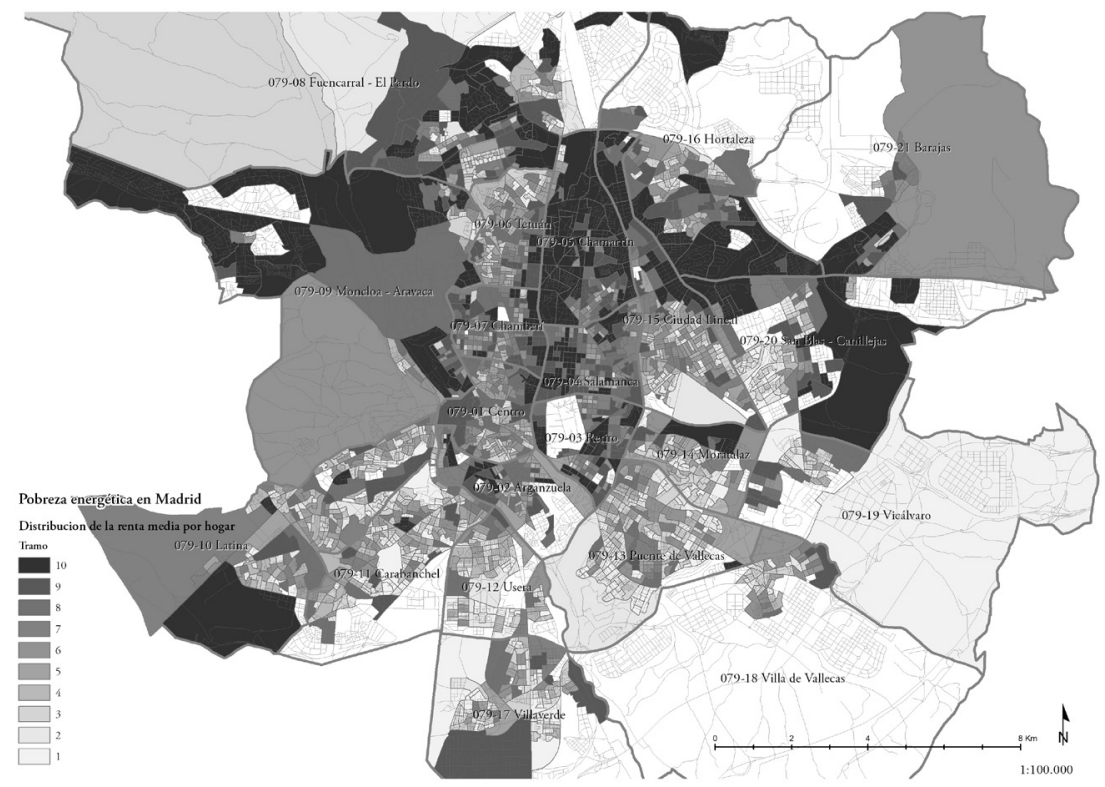

FUENTE ELABORACIÓN PROPIA CON DATOS DE EEA, 2017

Los valores de renta de este estudio aparecen detallados geográficamente a la escala de la sección censal. Ello permite una fotografía de la desigualdad urbana en cuestión de ingresos para los hogares de la ciudad de Madrid y establecer comparaciones con las estimaciones de demanda, consumo y precio de la energía realizados en el presente trabajo para la misma escala. En la figura 4 se ha representado el tramo de renta en el que se encuentra cada sección censal.

Finalmente, se calcula la relación entre el valor medio del gasto en servicios energéticos de las viviendas principales y la renta media disponible por hogar, para cada sección censal. Para introducir los datos se han utilizado los valores medios de cada clase de demanda energética. En lo que respecta a la etiqueta energética, para la clase A se ha utilizado el valor medio. Para la peor clase (G) se ha optado por utilizar el límite inferior, puesto que no es posible hacer estimaciones acerca del valor medio.

\section{Análisis de resultados}

La figura 5 representa el mapa de la pobreza energética de Madrid obtenido mediante la aplicación de la metodología descrita. Quedan representadas las secciones censales conforme a una clasificación en seis tramos y destacadas aquellas que superan el indicador de pobreza energética del 10\%. Para la representación en el mapa, se han considerado viviendas de tamaño medio y el precio por $\mathrm{kWh}$ necesario para satisfacer la demanda de calefacción correspondiente a la clase en demanda correspondiente a cada sección censal (tablas 3a y 3b). 
FIgURA 5 | Mapa de la pobreza energética en Madrid

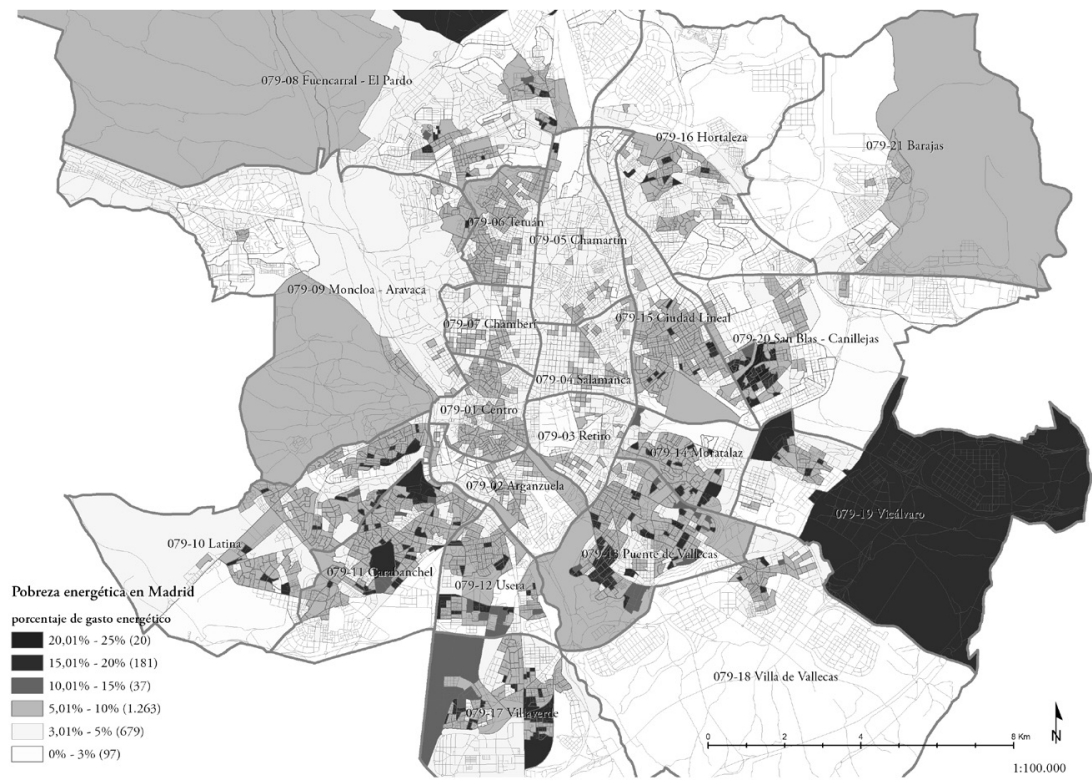

FUENTE ELABORACIÓN PROPIA, 2017

La calidad de la edificación, en general, es ineficiente en todas las secciones censales, debido a su antigüedad. En cuanto a la distribución de los hogares del tipo de combustible para calefacción (figura 2a y 2b), se comprueba que en 2001 la mayor implantación del gas natural era en las periferias más externas del municipio, desarrollos recientes que ya incorporan servicios de calefacción en la totalidad de las viviendas.

Se constata, a su vez, una mayor presencia de calefacción eléctrica en las zonas más vulnerables: en el centro de la ciudad, en el arco sur y en general en las zonas producidas en el periodo de 1940 a 1980, después de la Guerra Civil Española, gran parte de ellas de promoción pública. En el centro, la gran incidencia de la calefacción eléctrica se debe a un factor de antigüedad de la edificación, construida en gran parte con anterioridad a la puesta en marcha de los sistemas de calefacción por fluidos. A pesar de que la rehabilitación de la edificación en el centro ha incorporado sistemas de calefacción individual por gas natural en edificios de viviendas, esta implantación no supera aún el 50\% (figura 2a). No obstante, el área central no cae en problemas de pobreza energética por darse en ella, en general, rentas más altas que las de la periferia.

En el caso de las periferias, las políticas de poblados del periodo de 1940 a 1980 derivaron en la construcción de viviendas de calidad constructiva y prestaciones muy básicas que no incluían estos sistemas. Destaca el caso de San Blas, con grandes extensiones de edificación residencial con la menor implantación de calefacción por gas de la ciudad. Los edificios de viviendas del mismo periodo que se construían en las zonas de los primeros ensanches decimonónicos de la ciudad, más cercanas al centro, además de presentar mejores calidades constructivas en la envolvente 
(Oteiza et al., 2018), sí que suelen contar con servicio de calefacción, generalmente con sistemas centralizado, que en el momento de su construcción utilizaban la combustión continua con carbón. En el momento de este estudio los quemadores de carbón han sido mayormente sustituidos.

TABLA 5A | Porcentaje de los ingresos del hogar empleado para hacer frente al gasto con calefacción según tramo de renta y clase de demanda. Calefacción de gas

\begin{tabular}{|c|c|c|c|c|c|c|c|c|c|c|}
\hline & & & & \multicolumn{7}{|c|}{ COSTE TOTAL ENERGÍA GAS (€/AÑo) } \\
\hline \multicolumn{4}{|c|}{ RENTA (€/AÑo) } & 947,83 & 991,63 & 1097,89 & 1266,81 & 1516,89 & 1718,96 & 1752,73 \\
\hline MIN. & MEDIA & MAX. & TRAMO & $\mathbf{A}$ & B & C & D & $\mathbf{E}$ & $\mathbf{F}$ & G \\
\hline 0 & 5.600 & & RMI & $17 \%$ & $18 \%$ & $20 \%$ & $23 \%$ & $27 \%$ & $31 \%$ & $31 \%$ \\
\hline 0 & 9.173 & & SMI & $10 \%$ & $11 \%$ & $12 \%$ & $14 \%$ & $17 \%$ & $19 \%$ & $19 \%$ \\
\hline 0 & 10.660 & 21.320 & $\mathbf{I}$ & $9 \%$ & $9 \%$ & $10 \%$ & $12 \%$ & $14 \%$ & $16 \%$ & $16 \%$ \\
\hline 21.321 & 22.290 & 23.260 & 2 & $4 \%$ & $4 \%$ & $5 \%$ & $6 \%$ & $7 \%$ & $8 \%$ & $8 \%$ \\
\hline 23.261 & 24.302 & 25.343 & 3 & $4 \%$ & $4 \%$ & $5 \%$ & $5 \%$ & $6 \%$ & $7 \%$ & $7 \%$ \\
\hline 25.344 & 26.583 & 27.823 & 4 & $4 \%$ & $4 \%$ & $4 \%$ & $5 \%$ & $6 \%$ & $6 \%$ & $7 \%$ \\
\hline 27.824 & 29.353 & 30.883 & 5 & $3 \%$ & $3 \%$ & $4 \%$ & $4 \%$ & $5 \%$ & $6 \%$ & $6 \%$ \\
\hline 30.884 & 32.722 & 34.560 & 6 & $3 \%$ & $3 \%$ & $3 \%$ & $4 \%$ & $5 \%$ & $5 \%$ & $5 \%$ \\
\hline 34.561 & 36.800 & 39.040 & 7 & $3 \%$ & $3 \%$ & $3 \%$ & $3 \%$ & $4 \%$ & $5 \%$ & $5 \%$ \\
\hline 39.041 & 42.310 & 45.580 & 8 & $2 \%$ & $2 \%$ & $3 \%$ & $3 \%$ & $4 \%$ & $4 \%$ & $4 \%$ \\
\hline 45.581 & 51.365 & 57.150 & 9 & $2 \%$ & $2 \%$ & $2 \%$ & $2 \%$ & $3 \%$ & $3 \%$ & $3 \%$ \\
\hline 57.151 & & & IO & $2 \%$ & $2 \%$ & $2 \%$ & $2 \%$ & $3 \%$ & $3 \%$ & $3 \%$ \\
\hline
\end{tabular}

TABla 5B | Porcentaje de los ingresos del hogar empleado para hacer frente al gasto con calefacción según tramo de renta y clase de demanda. Calefacción por electricidad

\begin{tabular}{|c|c|c|c|c|c|c|c|c|c|c|}
\hline & & & & \multicolumn{7}{|c|}{ COSTE TOTAL ENERGÍA ELÉCTRICA (€/AÑo) } \\
\hline \multicolumn{4}{|c|}{ RENTA (€/AÑo) } & 930,58 & 1004,42 & 1183,65 & 1447,28 & 1930,65 & 2321,25 & 2386,58 \\
\hline MIN. & MEDIA & MAX. & TRAMO & $\mathbf{A}$ & B & C & $\mathbf{D}$ & $\mathbf{E}$ & $\mathbf{F}$ & G \\
\hline & 5.600 & & RMI & $17 \%$ & $18 \%$ & $21 \%$ & $26 \%$ & $34 \%$ & $41 \%$ & $43 \%$ \\
\hline & 9.173 & & SMI & $10 \%$ & $11 \%$ & $13 \%$ & $16 \%$ & $21 \%$ & $25 \%$ & $26 \%$ \\
\hline 0 & 10.660 & 21.320 & I & $9 \%$ & $9 \%$ & $11 \%$ & $14 \%$ & $18 \%$ & $22 \%$ & $22 \%$ \\
\hline 21.321 & 22.290 & 23.260 & 2 & $4 \%$ & $5 \%$ & $5 \%$ & $6 \%$ & $9 \%$ & $10 \%$ & $11 \%$ \\
\hline 23.261 & 24.302 & 25.343 & 3 & $4 \%$ & $4 \%$ & $5 \%$ & $6 \%$ & $8 \%$ & $10 \%$ & $10 \%$ \\
\hline 25.344 & 26.583 & 27.823 & 4 & $4 \%$ & $4 \%$ & $4 \%$ & $5 \%$ & $7 \%$ & $9 \%$ & $9 \%$ \\
\hline 27.824 & 29.353 & 30.883 & 5 & $3 \%$ & $3 \%$ & $4 \%$ & $5 \%$ & $7 \%$ & $8 \%$ & $8 \%$ \\
\hline 30.884 & 32.722 & 34.560 & 6 & $3 \%$ & $3 \%$ & $4 \%$ & $4 \%$ & $6 \%$ & $7 \%$ & $7 \%$ \\
\hline 34.561 & 36.800 & 39.040 & 7 & $3 \%$ & $3 \%$ & $3 \%$ & $4 \%$ & $5 \%$ & $6 \%$ & $6 \%$ \\
\hline 39.041 & 42.310 & 45.580 & 8 & $2 \%$ & $2 \%$ & $3 \%$ & $3 \%$ & $5 \%$ & $5 \%$ & $6 \%$ \\
\hline 45.581 & 51.365 & 57.150 & 9 & $2 \%$ & $2 \%$ & $2 \%$ & $3 \%$ & $4 \%$ & $5 \%$ & $5 \%$ \\
\hline 57.151 & & & Io & $2 \%$ & $2 \%$ & $2 \%$ & $3 \%$ & $3 \%$ & $4 \%$ & $4 \%$ \\
\hline
\end{tabular}

FUENTE ELABORACIÓN PROPIA, 2017 
En las tablas 5 a y 5 b se puede comprobar el grado de pobreza energética según la clasificación de la edificación. Se marcan en gris oscuro las situaciones en las que el coste de las facturas energéticas estaría superando el 10\% de los ingresos. Se puede comprobar que ni con la Renta Mínima de Inserción ni con el Salario Mínimo Interprofesional es posible mantener el hogar medio en situación de confort en ningún caso, ni siquiera en edificios de alta eficiencia energética. Para rentas situadas en el tramo 1, solamente quedan libres de situaciones de pobreza las viviendas más eficientes, de clasificación en demanda de calefacción А о в.

Además, en los casos en los que la fuente de energía es eléctrica (tabla 5b), la situación empeora y las viviendas de peor calificación ( $\mathrm{F}$ y G) que se encuentran en los tramos de renta 2 también entrarían en la clasificación de pobreza energética.

Se ha estimado a la vez el porcentaje de gasto considerando las rentas más bajas de cada tramo, comprobándose que no hay variaciones en las clases energéticas que entrarían en la situación de pobreza.

La rehabilitación energética para lograr la calidad térmica definida a partir de las exigencias del Código Técnico de la Edificación de España (clase D o superior) no garantiza la erradicación de la pobreza energética en las secciones censales clasificadas en el tramo de renta más bajo, ni para los hogares que ingresan el SMI o sobreviven gracias a la RMI.

\section{Discusión}

El consumo anual medido para una vivienda media situada en la zona continental de España es de $9.786 \mathrm{kWh}(35,3 \mathrm{GJ} /$ hogar$)$ en las viviendas en bloque y de 19.658kWh (70,8 GJ/hogar) en las unifamiliares (IDAE, 2011b). Su correspondencia en consumo de calefacción (55\% de la energía) sería de $5.382 \mathrm{kWh}$ en viviendas en bloque y de $10.812 \mathrm{kWh}$ en unifamiliares. Ambas cifras se sitúan por debajo de las estimaciones realizadas a partir de los valores medios de demanda para la vivienda en Madrid (tabla 2a).

El hecho de que las cifras de estimación de demanda energética, con base en las exigencias normativas, queden por debajo de los consumos medidos por el IDAE, podría indicar que al menos en las viviendas situadas en Madrid no se está satisfaciendo la exigencia en demanda, situándose por debajo del confort que prescribe la normativa. La pobreza energética es sin duda causante de este desfase, aunque no es el único factor. Se puede estar constatando que existe una privación voluntaria del confort por parte del usuario final, que prefiere ahorrar los costes derivados de su satisfacción para reducir gastos en el hogar y poder dedicar sus recursos a otros asuntos que considera más prioritarios. Esto sucedería principalmente en las rentas medias y bajas. Otra posible interpretación sería que la estimación de las demandas energéticas por el procedimiento normativo esté sobredimensionando las necesidades para quedar, por así decirlo, del lado de la seguridad. Esta circunstancia no tiene mayor importancia cuando se trata de clasificar las viviendas conforme a la escala de calificación energética, que está distribuida mediante el mismo procedimiento de cálculo, pero en el momento en el que se comparan con datos de renta se puede generar incertidumbre en los resultados. 
También hay que tener en cuenta que se están cruzando datos de diferentes fuentes y periodos: datos de la edificación (INE, 2001), datos de renta (2013) y precios de la energía en el momento de la consulta (2017). La validez de los resultados depende de que la situación de la edificación existente se haya mantenido desde el último censo de muestra universal, lo cual tiene sentido en los barrios que tienen una mayor necesidad de ser rehabilitados (generalmente los de la periferia). Además, para realizar este análisis se ha supuesto que los niveles de renta y su distribución espacial no han variado desde 2013 hasta el momento de la consulta de los precios de la energía, lo cual también tiene sentido si se tiene en cuenta el estancamiento económico en el que se encuentra España.

Los cálculos están realizados en todo momento para una vivienda de tamaño medio en Madrid (superficie de $80,76 \mathrm{~m}^{2}$ ), pero sería posible introducir en el análisis el tamaño de vivienda medio por sección censal, lo que haría el procedimiento más engorroso, pero tal vez más ajustado a la realidad. Previamente sería necesario analizar la variabilidad del tamaño medio de la vivienda para comprobar si difiere en los distintos barrios y distritos de la ciudad. Estudios recientes parecen indicar que no resulta destacable (Moya González, Fernández Salgado \& Escamilla Valencia, 2017) y por ello se ha optado por no introducir esta variable.

El análisis que aquí se presenta asume la definición del límite de pobreza energética asociado al nivel de renta. El trabajo de Bradshaw y Hutton (1983) que establece el límite de los gastos asociados a consumos energéticos en el 10\% de la renta, marca el origen de los indicadores objetivos basados en la dupla ingreso-gasto energético, actualizado posteriormente mediante el indicador de Hills (2012), el cual propone la definición Low Income-High Cost que incorpora el dato medio de la población (Sánchez-Guevara, Sanz Fernández \& Hernández Aja, 2015). Sin embargo, en Europa se ha desarrollado otro tipo de indicadores, por ejemplo indicadores subjetivos (Healy \& Clinch, 2004) o que incluyen el estado de conservación de la vivienda (Romero, Linares, López Otero, Lavandeira \& Pérez Alonso, 2014), que completan con información valiosa los estudios de pobreza energética (Heindl \& Schuessler, 2015). Resulta a su vez interesante el acercamiento de Moore (2012), que refiere problemas a la hora de definir la pobreza energética solamente a partir del porcentaje de los ingresos que suponen las facturas energéticas y el aumento del riesgo de mortalidad en condiciones climáticas extremas. En su estudio sugiere la incorporación de otros factores de vulnerabilidad en el análisis, incluyendo un enfoque estándar de presupuestos familiares que permita discriminar las rentas más bajas que en ningún caso van a poder hacer frente a sus costes energéticos, de las rentas altas con consumos energéticos disparados. La disponibilidad de los datos utilizados ha permitido realizar este estudio asumiendo la definición del 10\%; no obstante, sería interesante incorporar en el futuro otros enfoques e indicadores para enriquecer el análisis. 


\section{Conclusiones}

Se ha desarrollado un indicador objetivo de pobreza energética, basado en la renta de los hogares, la calidad térmica de la edificación residencial y el coste de la factura energética a escala urbana, que permite localizar áreas desfavorecidas en grandes ciudades. En estas zonas se concentran las circunstancias que dificultan mantener la calidad del ambiente interior de las viviendas dentro de rangos aceptables, los cuales se definen en la normativa vigente. Se ha propuesto una escala que permite cuantificar la severidad del problema y se ha aplicado la metodología en Madrid, obteniendo un mapa de la pobreza energética de la ciudad.

En el caso del municipio de Madrid, la pobreza energética resulta ser un fenómeno exclusivo de la periferia urbana. Se constata que se produce una acumulación de circunstancias causantes de la pobreza energética en los mismos barrios: demandas energéticas altas por mala calidad de la edificación, instalaciones de calefacción ineficientes mediante radiadores eléctricos y bajos niveles de renta.

La metodología permite estimar un valor aproximado del coste de la factura energética media de los hogares contenidos en cada área urbana. Los hogares contenidos en secciones censales clasificadas en el tramo de rentas más bajas (RMI, SMI y tramos 1 y 2), cuyas viviendas tienen clasificación energética de demanda de calefacción C, D, E, F y G, se encuentran todos en riesgo de pobreza energética.

La rehabilitación energética para lograr la calidad térmica definida a partir de las exigencias del Código Técnico de la Edificación de España para los edificios existentes (clase D o superior), no garantiza la erradicación de la pobreza energética en las secciones censales clasificadas en el tramo de renta más bajo, ni para SMI ni para RMI. La introducción de criterios de aplicación en esta norma para la inmensa casuística que comprenden los edificios existentes, tiene como objetivo evitar que un exceso de exigencia haga imposible su cumplimiento. Para los casos en los que no sea viable alcanzar el nivel de prestación establecido con carácter general, el Código Técnico de Edificación abre la puerta a la adopción de soluciones que permitan el mayor grado de adecuación posible. El criterio de flexibilidad introducido deriva en una debilidad para regular las desigualdades ocasionadas por la vulnerabilidad energética de la edificación existente. Esto apunta a la necesidad de desarrollar políticas públicas específicas para combatir la pobreza energética de manera complementaria a los planes para la rehabilitación energética de viviendas.

La fuente de energía es el factor que más influye en el precio del kilovatio hora $(\mathrm{kWh})$, pero también existen otros factores, como puede ser el tipo de tarifa que se contrate; y en un mercado recientemente liberalizado, como el espańol, influye la oferta de precios de cada empresa comercializadora. En el caso de la energía eléctrica, la potencia contratada que se suscriba también influye sensiblemente. La utilización de instalaciones de calefacción por energía eléctrica agrava el problema al resultar sensiblemente más elevada la factura energética resultante.

Se demuestra que la estructura tarifaria española deriva en un coste mayor por $\mathrm{kWh}$ para los consumos energéticos más bajos. Esta situación penaliza tanto a los hogares que incorporan medidas de mejora de la eficiencia energética, como a aquellos que se encuentran sufriendo situaciones de pobreza energética y carecen de 
recursos para hacer frente al costo de la energía suficiente para lograr el confort en sus viviendas. Esto se traduce en que las rentas más bajas, que intentan reducir gastos mediante el ahorro de combustible, reciben una mayor presión en la factura, a pesar de no satisfacer sus necesidades de confort. Además, esta situación desincentiva a aquellos hogares pertenecientes a los niveles de renta más altos, que podrían permitirse la inversión en la eficiencia energética pero no reciben estímulo, puesto que la repercusión de las mejoras en la factura energética resulta amortiguada.

La tarificación de precios de la energía hace además que, en el momento del presente estudio, la utilización del gas natural como combustible para la calefacción resulte sensiblemente más económico que la utilización de la calefacción eléctrica. Esta situación favorece la instalación de calderas de gas, normalmente individuales para cada vivienda, promoviéndose el agotamiento de combustibles fósiles no renovables y generando unas mayores emisiones de gases contaminantes y de efecto invernadero a la atmósfera, al menos en la ciudad y su entorno próximo, que además empeoran la calidad del aire exterior.

\section{Agradecimientos}

Al Ministerio de Economía, Industria y Competitividad del Gobierno de España por su financiación al proyecto HABITA-REs Nueva herramienta integrada de evaluación para áreas urbanas vulnerables. Hacia la autosuficiencia energética y a favor de un modelo de habitabilidad biosaludable. Ref. BIA20I7-8323 I-C2-I-R.

\section{Referencias bibliográficas}

Aksoezen, M., Daniel, M., Hassler, U. \& Kohler, N. (2015). Building age as an indicator for energy consumption. Energy and Buildings, (87), 74-86. https://doi.org/10.1016/j. enbuild.2014.10.074

Alonso, C., Martín-Consuegra, F. \& Lucas, P. (2013). Consideraciones socioeconómicas para la rehabilitación energética de edificios. Presentado en Jornadas internacionales de investigación en construcción: vivienda: pasado, presente y futuro: resúmenes y actas, Instituto de Ciencias de la Construcción "Eduardo Torroja", Madrid.

Ayuntamiento de Madrid (2017). Renta neta media de los hogares (Urban Audit). https://bit. ly/2AVhdDe

Bradshaw, J. \& Hutton, S. (1983). Social policy options and fuel poverty. Journal of Economic Psychology, 3(3-4) 249-266. https://doi.org/10.1016/0167-4870(83)90005-3

Código Técnico de Edificación (Сте) (2013). Documento Básico he Ahorro de energía. Versión publicada en el Boletín Oficial del Estado (вов) 12/09/2013, con corrección de errores del воE del 08/11/2013. http://www.arquitectura-tecnica.com/hit/Hit2016-2/ DBHE.pdf

Comisión Nacional de los Mercados y la Competencia (CNM) (2017). Comparador de Ofertas de Energia: Gas y Electricidad. https://comparadorofertasenergia.cnmc.es/comparador/ index.cfm?js $=1 \& e=\mathrm{N}$ 
Comité Económico y Social Europeo (CESE) (2011, 14 de febrero). Dictamen del Comité Económico y Social Europeo sobre el tema "La pobreza energética en el contexto de la liberalización y de la crisis económica" (Dictamen exploratorio). https://bit. ly/2QxEm9o

Comunidad Autónoma de Madrid (CAM) (2017). Orden 1003/2017, de 7 de junio, del Consejero de Politicas Sociales y Familia, por la que se aprueba el modelo normalizado de solicitud de prestación económica de Renta Minima de Inserción de la Comunidad de Madrid y se ordena su publicación, Consejería de Políticas Sociales y Familia $\$$ D. G. de Servicios Sociales e Integración Social. https://bit.ly/2UiMnxr

Cuchí, A., Sweatman, P. \& Rehabilitation Working Group “GTR” (2012). GTR's 2012 Report: A national perspective on Spain's building sector: action plan for a new housing sector. Madrid: Green Building Council España. https://core.ac.uk/download/pdf/41770648.pdf

Dalle, M., López Quero, M., Arregui Portillo, G., Avilés Palacios, C., Buendía García, L., Estrada López, B., ... Méndez Bértolo, E. (2010). La generación de empleo en la rehabilitación y modernización energética de edificios y viviendas. Instituto Sindical de Trabajo, Ambiente y Salud (ISTAS), 198. http://www.istas.ccoo.es/descargas/ empleo\%20en\%20rehabilitacion\%20edificios.pdf

Efinovatic \& Centro Nacional de Energías Renovables (CENER) (2016, abril). Guía IDAE: Manual de usuario de calificación energética de edificios existentes $C E^{3} X$. Madrid: Efinovatic \& CENER. https://es.scribd.com/document/351917717/1-Manual-de-usuario-CE-X

European Commission \& Directorate-General for Energy (2010). EU energy trends to 2030: update 2009. Luxembourg: EUR-OP.

European Environment Agency (2017). Urban Audit [External Data Reference]. https://www. eea.europa.eu/data-and-maps/data/external/urban-audit-database

European Union (2017). Methodological manual on city statistics. 2017 edition. Luxembourg: Publications Office of the European Union. https://bit.ly/2AT5ogI

García, L. A. R. (2006). The liberalisation of the Spanish gas market. Energy Policy, 34(13), 1630-1644. https://doi.org/10.1016/j.enpol.2004.12.013

Gómez Muñoz, G. (2014). Método de análisis diacrónico para la intervención en el alojamiento con criterios ecológicos. El caso de Madrid 1940-2100. Tesis Doctoral. E.T.s. Arquitectura (Universidad Politécnica de Madrid). http://oa.upm.es/30897/

Healy, J. D. (2003). Excess winter mortality in Europe: a cross country analysis identifying key risk factors. Journal of epidemiology and community health, 57(10), 784-789. https:// jech.bmj.com/content/57/10/784

Healy, J. D. \& Clinch, J. P. (2004). Quantifying the severity of fuel poverty, its relationship with poor housing and reasons for non-investment in energy-saving measures in Ireland. Energy Policy, 32(2), 207-220. https://doi.org/10.1016/S0301-4215(02)00265-3

Heindl, P. \& Schuessler, R. (2015). Dynamic properties of energy affordability measures. Energy Policy, 86, 123-132. https://doi.org/10.1016/j.enpol.2015.06.044

Hills, J. (2012). Getting the measure of fuel poverty: Final Report of the Fuel Poverty Review. Case Report 72. London: Centre for Analysis of Social Exclusion (CASE), The London School of Economics and Political Science, Department of Energy and Climate Change (DECC). http://sticerd.lse.ac.uk/dps/case/cr/CASEreport72.pdf

Instituto Nacional de Estadística (INE) (2001). Censo de Población y Viviendas de 2001. http:// www.ine.es/censo2001/ 
Instituto Nacional de Estadística (INE) (2011). Censo de Población y Viviendas de 2011. http:// www.ine.es/censos2011_datos/cen11_datos_inicio.htm

Instituto para la Diversificación y Ahorro de la Energía (IDAE) (2011a). Escala de calificación energética para edificios existentes. Madrid: Ministerio de Fomento / Ministerio de Industria, Comercio y Turismo. https://bit.ly/2rmUn39

Instituto para la Diversificación y Ahorro de la Energía (IDAE) (2011b). Proyecto SECH-SPAHOUSEC. Análisis del consumo energético del sector residencial en España. Informe Final. Madrid: IDAE / Eurostat - European Commission / Ministerio de Industria, Energía y Turismo. https://bit.ly/2zH7UXK

Lelkes, O. \& Zólyomi, E. (2010). Housing quality deficiencies and the link to income in the $E U$. Policy Brief 3/2010. Viena: European Centre. https://www.euro.centre.org/ publications/detail/383

Lelkes, O. \& Zólyomi, E. (2015). Improvement during crisis years? Poverty and housing conditions across the EU, 2007-2012. Policy Brief 9/2015. Viena: European Centre. https://www. euro.centre.org/publications/detail/418

Liddell, C. \& Morris, C. (2010). Fuel poverty and human health: A review of recent evidence. Energy Policy, 38(6), 2.987-2997. https://doi.org/10.1016/j.enpol.2010.01.037

Martín-Consuegra, F., Hernández Aja, A., Oteiza, I. \& Alonso, C. (2016). Energy needs and vulnerability estimation at an urban scale for residential neighbourhoods heating in Madrid (Spain). En Proceedings of PLEA 2016 Los Angeles - 32th International Conference on Passive and Low Energy Architecture (Vol. 3, pp. 1.413-1.419). Los Ángeles, CA. EE.UU. http://oa.upm.es/44264/1/1109-PLEA2016_Martin-Consuegra.pdf

Ministerio de Empleo y Seguridad Social (EmpleoGob) (2015). Real Decreto 1171/2015, de 29 de diciembre, por el que se fija el salario mínimo interprofesional para 2016. http:// noticias.juridicas.com/base_datos/Fiscal/565513-rd-1171-2015-de-29-dic-salariominimo-interprofesional-para-2016.html

Moore, R. (2012). Definitions of fuel poverty: Implications for policy. Energy Policy, 49, 19-26. https://doi.org/10.1016/j.enpol.2012.01.057

Moya González, L., Fernández Salgado, C. \& Escamilla Valencia, F. (2017). Evolución del tamaño de la vivienda de promoción pública y su comparación con el resto del parque residencial construido en Madrid entre 1940-2010. Informes de la Construcción, 69(545), 178. https://bit.ly/2SCQfaX

Oteiza, I., Alonso, C., Martín-Consuegra, F. \& Buldón, A. (2016). Characterization of social housing in Madrid (1939-1979) for its energy efficiency improvement. Presentado en EESAP7 / The European Conference on Energy Efficiency and Sustainability in Architecture and Planning.

Oteiza, I., Alonso, C., Martín-Consuegra, F., González-Moya, M. \& Monjo, J. (2015). Energy retrofitting for social housing by improving the building envelope: Madrid, 19391979. En P. Mercader-Moyano (ed.), The sustainable renovation of buildings and neighbourhoods (pp. 3-32). Bentham eBooks

Oteiza, I., Alonso, C., Martín-Consuegra, F., Monjo, J., González Moya, M. \& Buldón, A. (2018). La envolvente energética de la vivienda social. El caso de Madrid en el periodo 1939-1979. Monografía No. 428. Instituto Eduardo Torroja de Ciencias de la Construcción. Consejo Superior de Investigaciones Científicas, Madrid. 
Parlamento Europeo \& Consejo de la Unión Europea (2012). Directiva 2012/27/uE del Parlamento Europeo y del Consejo, de 25 de octubre de 2012, relativa a la eficiencia energética, por la que se modifican las Directivas 2009/125/CE y 2010/30/UE, y por la que se derogan las Directivas 2004/8/CE y 2006/32/CE Texto pertinente a efectos del EEE. Diario Oficial de la Unión Europea. https://www.boe.es/doue/2012/315/L0000100056.pdf

Romero, J. C., Linares, P., López Otero, X., Lavandeira, X. \& Pérez Alonso, A. (2014). Pobreza energética en España. Análisis económico y propuestas de actuación. Vigo: Economics for Energy.

Sánchez-GuevaraSánchez, C. (2015). Propuesta metodológica de evaluación de la pobreza energética en España: indicadores para la rehabilitación de viviendas. Tesis doctoral. Escuela Técnica Superior de Arquitectura de Madrid (ETS), Universidad Politécnica de Madrid (UPM). http://oa.upm.es/39928/

Sánchez-Guevara, C., Sanz Fernández, A. \& Hernández Aja, A. (2015). Income, energy expenditure and housing in Madrid: retrofitting policy implications. Building Research \& Information, 43(6), 737-749. https://doi.org/10.1080/09613218.2014.984573

Song, J., Li, H. \& Wallin, F. (2017). Cost comparison between district heating and alternatives during the price model restructuring process. Energy Procedia, 105(Supplement c), 3.922-3.927. https://doi.org/10.1016/j.egypro.2017.03.813

Temes, R. R. (2014). Valoración de la vulnerabilidad integral en las áreas residenciales de Madrid. EURE - Revista de Estudios Urbano Regionales, 40(119). http://www.eure.cl/ index.php/eure/article/view/344

Thomson, H. \& Snell, C. (2013). Quantifying the prevalence of fuel poverty across the European Union. Energy Policy, 52, 563-572. https://doi.org/10.1016/j.enpol.2012.10.009

Tirado, S., Jiménez Meneses, L., López Fernández, J. L., Perero Van Hove, E., Irigoyen Hidalgo, V. \& Savary, P. (2016). Pobreza, vulnerabilidad y desigualdad energética. Nuevos enfoques de análisis. Madrid: Asociación de Ciencias Ambientales.

www [World Wildlife Fund / Foro Mundial por la Naturaleza], Velázquez, I., Verdaguer, C., Sanz, A- \& Gómez, G. (2017). Rehabilitación energética de viviendas. Cómo mejorar la calidad de vida y combatir el cambio climático. Madrid: wwF. https://bit.ly/2rJoYWV 